\title{
CYCLIC PROPERTIES OF TRIANGULAR GRID GRAPHS
}

\author{
Yury Orlovich ${ }^{1}$, Valery Gordon ${ }^{2}$, Frank Werner ${ }^{3}$ \\ ${ }^{1}$ Institute of Mathematics, National Academy of Sciences of Belarus, 11 Surganova Str., \\ 220072 Minsk, Belarus; e-mail: orlovich@im.bas-net.by \\ ${ }^{2}$ United Institute of Informatics Problems, National Academy of Sciences of Belarus, \\ 6 Surganova Str., 220012 Minsk, Belarus; e-mail: gordon@newman.bas-net.by \\ ${ }^{3}$ Institute of Mathematical Optimization, Otto-von-Guericke-University of Magdeburg, \\ Universitätsplatz 2, 39106 Magdeburg, Germany; \\ e-mail:frank.werner@mathematik.uni-magdeburg.de
}

\begin{abstract}
It is known that all 2-connected, linearly convex triangular grid graphs, with only one exception, are hamiltonian (Reay and Zamfirescu, 2000). In the paper, it is shown that this result holds for a wider class of connected, locally connected triangular grid graphs and, with more exceptions, even for some general class of graphs. It is also shown that the HAMILTONIAN CYCLE problem is NP-complete for triangular grid graphs. Copyright (C) 2006 IFAC
\end{abstract}

Keywords: Combinatorial mathematics, Graph theory, Hamiltonian graph, Triangular grid graph, Local connectivity, NP-completeness.

\section{INTRODUCTION}

Scheduling with precedence constraints is closely related to the investigation of specific graph properties. For instance, finding a minimum hamiltonian path in a weighted graph is known to be equivalent to minimizing the makespan in a single machine scheduling problem with setups. In the paper, cyclic properties (hamiltonicity and fully cyclic extendability) of finite induced subgraphs of an infinite graph associated with the two-dimensional triangular grid (called triangular grid graphs) are considered. An application of these properties arises in telecommunications and computer vision (problems of determining the shape of an object represented by a cluster of points on a grid), in molecular biology (protein folding), and in configurational statistics of polymers (Agarwala, et al., 1997; Lua, et al., 2004). Cyclic properties of triangular grid graphs can also be used in the design of cellular networks since these networks are generally modelled as induced subgraphs of the infinite two-dimensional triangular grid (Havet, 2001).

For graph-theoretic terminology not defined here, the reader is referred to (Bondy and Murty, 1976). Let $G$ be a graph with the vertex set $V(G)$ and the edge set $E(G)$. A path in $G$ with the end vertices $u$ and $v$ is called a $(u, v)$-path. Graph $G$ is connected if there exists a $(u, v)$-path for any vertices $u$ and $v$ of $G$.
Graph $G$ is $k$-connected $(k \geq 2)$ if any two distinct vertices $u$ and $v$ of $G$ are connected by at least $k$ internally-disjoint $(u, v)$-paths. For a vertex $u$ of $G$, the neighborhood $N(u)$ of $u$ is the set of all vertices adjacent to $u$. For a subset of vertices $X \subseteq V(G)$, the subgraph of $G$ induced by $X$ is denoted by $G(X)$. A vertex $u$ of $G$ is said to be locally connected if $G(N(u))$ is connected. $G$ is called locally connected if each vertex of $G$ is locally connected.

Graph $G$ is hamiltonian if $G$ has a hamiltonian cycle, i.e. a cycle containing all vertices of $G$. Similarly, a path that contains all vertices of $G$ is a hamiltonian path. As usual, $P_{k}$ and $C_{k}$ denote the path and the cycle on $k$ vertices, respectively. In particular, $C_{3}$ is a triangle. A cycle $C$ in a graph $G$ is extendable if there exists a cycle $C^{\prime}$ in $G$ such that $V(C) \subset V\left(C^{\prime}\right)$ and $\left|V\left(C^{\prime}\right)\right|=|V(C)|+1$. A connected graph $G$ is fully cyclic extendable if every vertex of $G$ is on a triangle and every nonhamiltonian cycle is extendable. Clearly, any fully cycle extendable graph is hamiltonian.

The infinite graph $T^{\infty}$ associated with the twodimensional triangular grid is defined as follows. The vertices of $T^{\infty}$ are points with cartesian coordinates $(x+y / 2, y \sqrt{3} / 2)$ for integers $x$ and $y$. Two vertices of $T^{\infty}$ are adjacent if and only if the Euclidean distance 
between them is equal to 1 . Graph $T^{\infty}$ is considered as a geometric graph, i.e. a graph drawn in the plane such that every of its edges is a closed straight-line segment and the edges intersect only at their ends (see Fig.1).

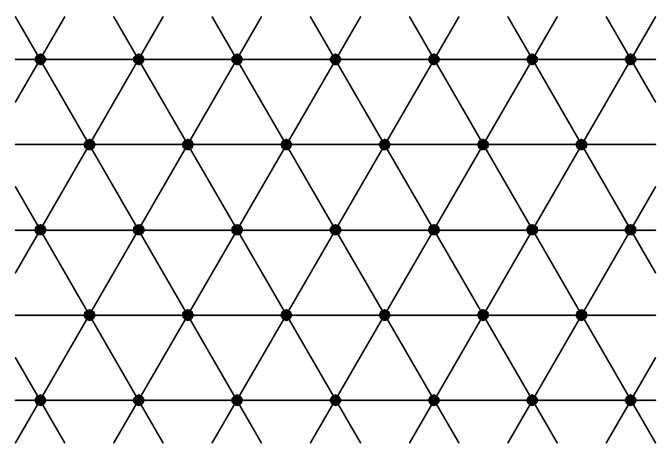

Fig. 1. A fragment of graph $T^{\infty}$.

A triangular grid graph (or $T^{*}$-graph) is a finite induced subgraph of $T^{\infty}$. A triangular grid graph $\mathrm{G}$ is linearly convex if, for every line $l$ which contains an edge of $T^{\infty}$, the intersection of $l$ and $G$ is either a line segment (a path in $G$ ), or a point (a vertex in $G$ ), or empty. For example, the $T^{*}$-graph $G$ (with three components including an isolated vertex $w$ ) shown in Fig. 2 is linearly convex even though $G$ has vertices $u$ and $v$ whose midpoint $z$ is a vertex of $T^{\infty}$ but not of $G$. In Fig. 2, dark points correspond to the vertices of $T^{\infty}$.

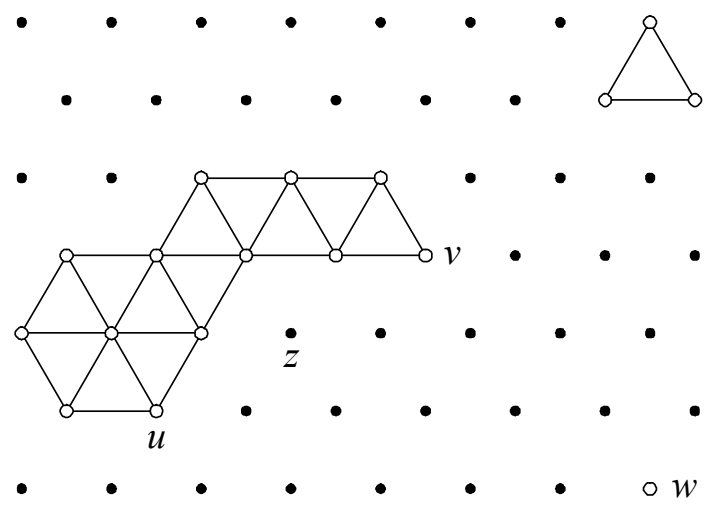

Fig. 2. A linearly convex triangular grid graph.

It is well-known that the problem of deciding whether a given graph is hamiltonian, is NPcomplete, and it is natural to look for conditions for the existence of a hamiltonian cycle for special classes of graphs. Our goal here is to determine such conditions for triangular grid graphs and for a wider class of graphs with the special structure of local connectivity.

The concept of local connectivity of a graph has been introduced by Chartrand and Pippert (1974). Oberly and Sumner (1979) have shown that a connected, locally connected claw-free graph $G$ on $n \geq 3$ vertices is hamiltonian (a graph is claw-free if it has no induced subgraph isomorphic to the complete bipartite graph $K_{1,3}$ ). Clark (1981) has proved that, under the Oberly - Sumner's conditions, $G$ is vertex pancyclic (i.e., every vertex of $G$ is on cycles of length $3,4, \ldots, n)$. Later, Hendry $(1989,1990)$ has introduced the concept of cycle extendability and strengthened Clark's result showing that, under the same conditions, $G$ is fully cycle extendable. Hendry (1989) has shown that connected, locally connected graphs in which the maximum and minimum degrees differ by at most one and do not exceed five are fully cycle extendable. Some further strengthenings of these results can be found in the survey by Faudree et al. (1997).

Below it is shown that any 2-connected, linearly convex triangular grid graph is locally connected. Cyclic properties (fully cycle extendability) of locally connected graphs with a special neighborhood structure are considered. In particular, it is established that a connected, locally connected triangular grid graph with $n \geq 3$ vertices is either fully cycle extendable or isomorphic to a special graph on thirteen vertices. It is also shown that the HAMILTONIAN CYCLE problem is NP-complete for triangular grid graphs.

\section{LOCAL CONNECTIVITY AND CYCLE EXTENDABILITY}

Reay and Zamfirescu (2000) have shown that all 2connected, linearly convex $T^{*}$-graphs, with only one exception, are hamiltonian. The only exception is a 13-vertices graph $D$ which is the linearly-convex hull of the Star of David; this graph is 2-connected and linearly convex but not hamiltonian (see Fig. 3).

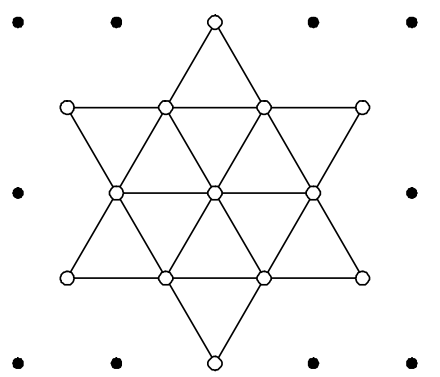

Fig. 3. Graph D.

An interrelation between classes of 2-connected, linearly convex $T^{*}$-graphs (or $T$-graphs in terminology of Reay and Zamfirescu, 2000) and locally connected $T^{*}$-graphs is established in the following theorem.

Theorem 1. Let $G$ be a 2-connected $T^{*}$-graph. If $G$ is linearly convex, then $G$ is locally connected.

Proof. The proof will be done by contradiction. We first introduce some useful additional notation. Note that each vertex $u$ of graph $T^{\infty}$ has six neighbors denoted as R (right), L (left), UR (up-right), DL (down-left), DR (down-right) and UL (up-left), respectively (see Fig. 4). For example, the notation $v=\operatorname{UR}(u)$ means that vertex $v$ is the up-right neighbor of vertex $u$. 


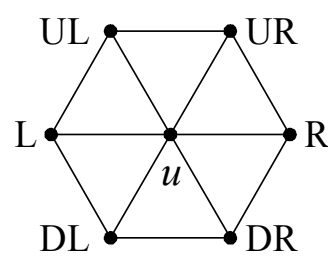

Fig. 4. Neighbors of the vertex $u$.

Let $G$ be a 2-connected, linearly convex triangular grid graph. Assume, to the contrary, that $G$ contains a vertex $u$ which is not locally connected. Note that $\operatorname{deg} u \leq 4$ (otherwise $G(N(u)$ ) is connected and isomorphic to $P_{5}$ if $\operatorname{deg} u=5$ or to $C_{6}$ if $\operatorname{deg} u=6$ ). On the other hand, the 2-connectedness of $G$ implies $\operatorname{deg} u \geq 2$. Consider the three possible cases for the degree of $u$.

Case 1. $\operatorname{deg} u=2$.

Let $N(u)=\{v, w\}$. By symmetry, we need only consider two subcases: $v=\operatorname{UR}(u), \quad w=\operatorname{DL}(u)$ (Fig. 5a), and $v=\operatorname{UR}(u), \quad w=\operatorname{DR}(u) \quad$ (Fig. 5b). Since $G$ is 2-connected, there exists a $(v, w)$-path $P$ in $G$ with internal vertices different from $u$. Let $l$ be a line which contains the edge $u \mathrm{R}(u)$ of $T^{\infty}$. Then the intersection of $l$ and $G$ contains vertex $u$ as an isolated vertex (since $\mathrm{L}(u)$ and $\mathrm{R}(u)$ are not in $G$ ) and at least one vertex of $P$. This contradicts the condition that $G$ is linearly convex.

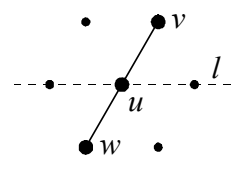

a)

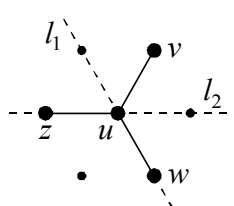

d)

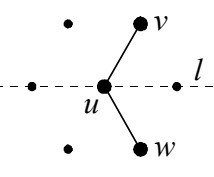

b)

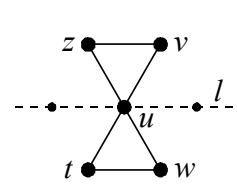

e)

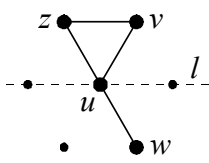

c)

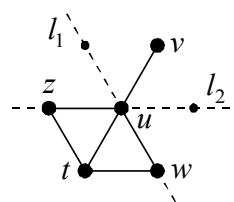

f)
Fig. 5. Cases $1-3$.

Case 2. $\operatorname{deg} u=3$.

Let $N(u)=\{v, w, z\}$. By symmetry, there are two subcases: $\quad v=\operatorname{UR}(u), \quad w=\operatorname{DR}(u), \quad z=\operatorname{UL}(u)$ (Fig. 5c), and $v=\mathrm{UR}(u), \quad w=\mathrm{DR}(u), \quad z=\mathrm{L}(u)$ (Fig. 5d). In the first subcase, the proof is similar to the proof in Case 1. Consider the second subcase. Since $G$ is 2 -connected, there exists a $(v, w)$-path $P$ in $G$ with internal vertices different from $u$. Let $l_{1}$ be a line which contains the edge $u w$ of $T^{\infty}$, and $l_{2}$ be a line which contains the edge $u z$ of $T^{\infty}$. Obviously, the intersection of $l_{1}$ and $G$ contains the edge $u w$ and does not contain $\operatorname{UL}(u)$, and the intersection of $l_{2}$ and $G$ contains the edge $u z$ and does not contain $\mathrm{R}(u)$. On the other hand, the intersection of these lines and graph $G$ contains at least one vertex of path $P$ either on the ray $l^{\prime}$ or on the ray $l^{\prime \prime}$. Here $l^{\prime}$ and $l^{\prime \prime}$ are the rays (parts of the lines $l_{1}$ and $l_{2}$ ) which start from $u$, and pass $\operatorname{UL}(u)$ and $\mathrm{R}(u)$, respectively. Hence, we arrive at a contradiction to the condition that $G$ is linearly convex.

Case 3. $\operatorname{deg} u=4$.

Let $N(u)=\{v, w, z, t\}$. By symmetry, there are two subcases: $\quad v=\operatorname{UR}(u), \quad w=\operatorname{DR}(u), \quad t=\operatorname{DL}(u)$, $z=\mathrm{UL}(u)$ (Fig. 5e), and $v=\mathrm{UR}(u), w=\mathrm{DR}(u)$, $t=\mathrm{DL}(u), z=\mathrm{L}(u)$ (Fig. 5f). The proof is similar to the proof in Case 2. This completes the proof of the theorem.

Note that the converse assertion to Theorem 1 is not true and an example can be found in Fig. 6. This example shows a connected, locally connected $T^{*}$ graph which is not linearly convex: the intersection of the graph and the dashed line, which contains edges of $T^{\infty}$, is the union of a line segment (the edge $v w$ of the graph) and a point (the isolated vertex $u$ of the graph).

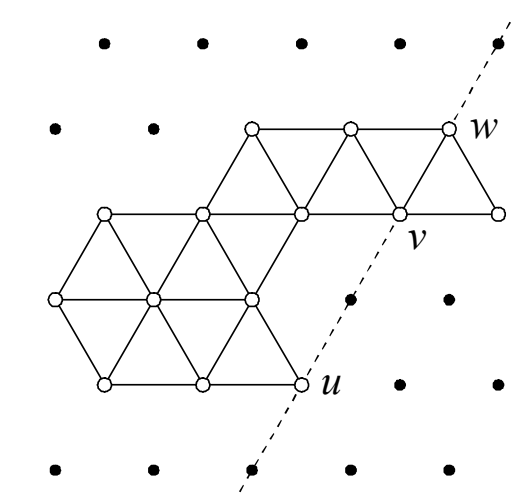

Fig. 6. A locally connected, but not linearly convex triangular grid graph.

Thus, the example in Fig. 6 and Theorem 1 show that 2-connected, linearly convex $T^{*}$-graphs form a proper subclass of the class of connected, locally connected $T^{*}$-graphs. Note that the graphs of this class (except an isolated vertex and a complete graph on two vertices) are also 2-connected due to a wellknown observation of Chartrand and Pippert (1974) that a connected, locally $k$-connected graph is $(k+1)$-connected.

It is easy to see that the neighborhood of any vertex of a connected, locally connected $T^{*}$-graph on $n \geq 3$ vertices induces a subgraph which is isomorphic to one of the following five graphs: $P_{2}, P_{3}, P_{4}, P_{5}$, and $C_{6}$. Consider class $M$ of all connected graphs $G$ with the property that for any vertex $u \in V(G)$ the following inclusion holds:

$$
G(N(u)) \in\left\{P_{2}, P_{3}, C_{3}, P_{4}, C_{4}, P_{5}, C_{5}, C_{6}\right\} .
$$


It is clear that connected, locally connected $T^{*}$ graphs on $n \geq 3$ vertices and, consequently, 2connected, linearly convex $T^{*}$-graphs belong to the class $M$. Cyclic properties of the graphs from the class $M$ are determined by the following theorem.

Theorem 2. If $G \in M$, then $G$ is either fully cycle extendable or isomorphic to $D$ or to one of the graphs $G_{1}, G_{2}$ (see Fig. 7).
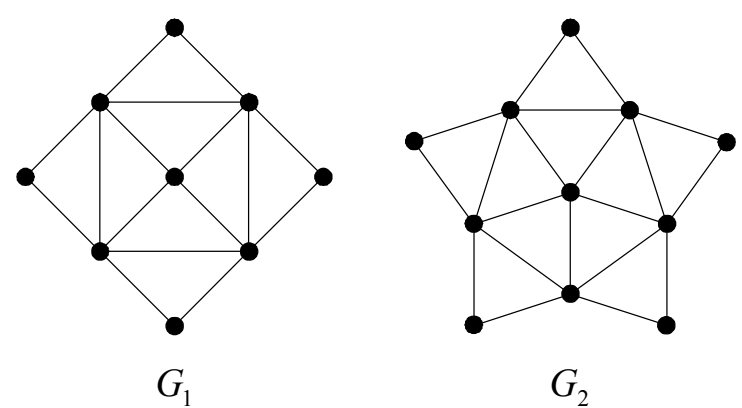

Fig. 7. Graphs $G_{1}$ and $G_{2}$.

The scheme of the proof is the following. Note that every vertex of $G$ has a degree of at least 2 and lies on a triangle of $G$ since $G$ is locally connected. Now suppose that $G$ is not fully cycle extendable, i.e. there exists a nonextendable, nonhamiltonian cycle $C=u_{1} u_{2} \ldots u_{k} u_{1}$ on $k<|V(G)|$ vertices in $G$. Since $G$ is connected, there exists a vertex $x$ not on $C$ which is adjacent to a vertex lying on $C$. Without loss of generality, let $u_{1}$ be a vertex on $C$ adjacent to $x$. Then $3 \leq \operatorname{deg} u_{1} \leq 6$. Via the series of claims $1-8$, one proceeds towards a contradiction with the nonextendability of the cycle $C$ or obtain that $G$ is isomorphic to one of the graphs $D, G_{1}$, or $G_{2}$.

Claim 1. Inequality $\operatorname{deg} u_{1} \geq 4$ holds.

Claim 2. All neighbors of $u_{1}$ except $x$ are on $C$.

Claim 3. Relation $\operatorname{deg} u_{1} \neq 4$ holds.

Claim 4. If $\operatorname{deg} x \geq 3$, then the subgraphs $G\left(\left\{u_{1}, u_{3}, x\right\}\right)$ and $G\left(\left\{u_{1}, u_{k-1}, x\right\}\right)$ are triangles in $G$.

Claim 5. Relation $\operatorname{deg} u_{1} \neq 6$ holds.

Claim 6. Relations $\operatorname{deg} u_{1}=5$ and $2 \leq \operatorname{deg} x \leq 6$ hold.

Claim 7. If $\operatorname{deg} u_{1}=5$ and $\operatorname{deg} x=2$, then either $C$ is an extendable cycle or graph $G$ is isomorphic to one of the graphs $D, G_{1}$, or $G_{2}$.

Claim 8. If $\operatorname{deg} u_{1}=5$ and $3 \leq \operatorname{deg} x \leq 6$, then $C$ is an extendable cycle.

Thus, according to Claims 7 and 8 one arrives at a contradiction with nonextendability of $C$ or obtain that $G$ is isomorphic to one of the graphs $D, G_{1}$, or $G_{2}$. This completes the scheme of the proof of Theorem 2 .
Note that the graphs $G_{1}$ and $G_{2}$ are not triangular grid graphs unlike the graph $D$. The following two corollaries are immediate consequences of Theorem 2.

Corollary 1. Let $G$ be a connected, locally connected $T^{*}$-graph on $n \geq 3$ vertices. Then $G$ is either fully cycle extendable or isomorphic to the graph $D$.

Corollary 2. Let $G$ be a 2-connected, linearly convex $T^{*}$-graph. Then $G$ is either fully cycle extendable or isomorphic to the graph $D$.

Thus, the main result of (Reay and Zamfirescu, 2000) on the hamiltonicity of 2-connected, linearly convex $T^{*}$-graphs directly follows from Corollary 2 .

\section{HAMILTONICITY OF TRIANGULAR GRID GRAPHS}

Consider the following well-known decision problem.

\section{HAMILTONIAN CYCLE}

Instance: A graph $G$.

Question: Is $G$ hamiltonian?

The problem is NP-complete for general graphs and remains difficult for graphs of many special classes (Garey and Johnson, 1979). Among them, there are bipartite graphs, line graphs, 3-connected cubic (i.e., 3-regular) planar graphs, maximal planar graphs, and others. In (Itai et al., 1982), it has been proved that the HAMILTONIAN CYCLE problem is NPcomplete for grid graphs (a grid graph is a finite induced subgraph of the two-dimensional rectangular grid). Notice that grid graphs are not a subclass of triangular grid graphs: these classes of graphs have common elements but in general they are distinct. The idea of (Itai et al., 1982) is used for proving the following theorem.

Theorem 3. The problem HAMILTONIAN CYCLE is NP-complete for $T^{*}$-graphs.

The proof is based on a polynomial-time reduction from the following NP-complete problem: HAMILTONIAN CYCLE for cubic planar bipartite graphs (Plesnik, 1983).

Note that Corollary 1 implies the polynomial solvability of the HAMILTONIAN CYCLE problem for locally connected $T^{*}$-graphs. Moreover, the following statements hold.

Theorem 4. Let $G \in M$ and $G$ be not isomorphic to $D, G_{1}$, and $G_{2}$. If $C$ is a cycle of length $k$ in $G$ and $3 \leq k<|V(G)|$, then a cycle $C^{\prime}$ of length $k+1$ such that $V(C) \subset V\left(C^{\prime}\right)$ can be found in polynomial time.

Corollary 3. A hamiltonian cycle in a connected, locally connected $T^{*}$-graph (not isomorphic to $D$ ) can be found in polynomial time. 


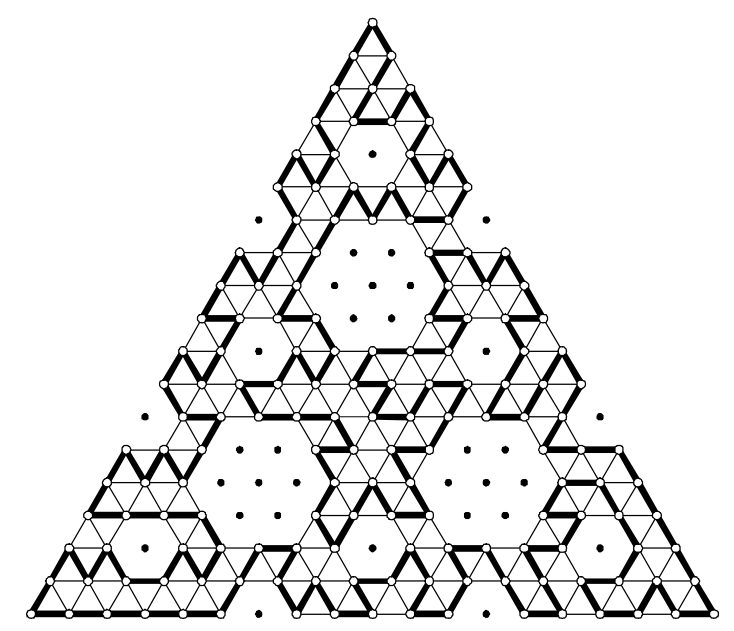

Fig. 8. A locally connected $T^{*}$-graph and one of its hamiltonian cycles.

An example of a locally connected $T^{*}$-graph with one of its hamiltonian cycles (bold lined) is shown in Fig. 8. This graph is not linearly convex and contains holes. Note that a polynomial algorithm for finding a hamiltonian cycle in a grid graph is known only in case when the graph does not contain holes (Lenhart and Umans, 1997).

Finally consider the following problem.

HAMILTONIAN $(u, v)$-PATH

Instance: A graph $G$.

Question: Does $G$ contain a hamiltonian $(u, v)$-path?

The following statement can be proved using Theorem 3.

Theorem 5. The problem HAMILTONIAN $(u, v)$ PATH is NP-complete for $T^{*}$-graphs.

\section{CONCLUSION}

In the paper, the result of Reay and Zamfirescu (2000), who proved that all 2-connected, linearly convex triangular grid graphs (with only one exception of graph $D$ ) are hamiltonian, is extended to a much wider class, where the HAMILTONIAN CYCLE problem can be solved in polynomial time. This class contains all locally connected triangular grid graphs and all linearly convex triangular grid graphs as proper subclasses. It is also shown that the HAMILTONIAN CYCLE problem is NP-complete for general triangular grid graphs.

\section{ACKNOWLEDGEMENTS}

This work is partially supported by INTAS (Project 03-51-5501) and, for the second author, by ISTC (Project B-986).

\section{REFERENCES}

Agarwala, R., S. Batzoglou, V. Dancík, S.E. Decatur, M. Farach, S. Hannenhalli and S. Skiena (1997).
Local rules for protein folding on a triangular lattice and generalized hydrophobicity in the HP model. Journal of Computational Biology, 4, 275-296.

Bondy, J.A. and U.S.R. Murty (1976). Graph Theory with Applications. Elsevier, New York.

Chartrand, G. and R. Pippert (1974). Locally connected graphs. Casopis pro Pestování Matematiky, 99, 158-163.

Clark, L. (1981). Hamiltonian properties of connected locally connected graphs. Congressus Numerantium, 32, 199-204.

Faudree, R.J., E. Flandrin and Z. Ryjácek (1997). Claw-free graphs - A survey. Discrete Mathematics, 164, 87-147.

Garey, M.R. and D.S. Johnson (1979). Computers and intractability. A guide to the theory of NPcompleteness. Freeman and Co., San Francisco.

Havet, F. (2001). Channel assignment and multicoloring of the induced subgraphs of the triangular lattice. Discrete Mathematics, 233, 219-231.

Hendry, G.R.T. (1989). A strengthening of Kikust's theorem. Journal of Graph Theory, 13, 257-260.

Hendry, G.R.T. (1990). Extending cycles in graphs, Discrete Mathematics, 85, 59-72.

Itai, A., C.H. Papadimitriou and J.L. Szwarcfiter (1982). Hamiltonian paths in grid graphs. SIAM Journal on Computing, 11, 676-686.

Lenhart, W. and C. Umans (1997). Hamiltonian cycles in solid grid graphs. In: Proceedings 38th Annual Symposium on Foundations of Computer Science (FOCS '97), 496-505. IEEE Computer Society, Washington, DC.

Lua, R., A.L. Borovinskiy and A.Yu. Grosberg (2004). Fractal and statistical properties of large compact polymers: a computational study. Polymer, 45, 717-731.

Oberly, D.J. and D.P. Sumner (1979). Every connected, locally connected nontrivial graph with no induced claw is Hamiltonian. Journal of Graph Theory, 3, 351-356.

Plesnik, J. (1983). The NP-completeness of the Hamiltonian cycle problem in bipartite cubic planar graphs. Acta Mathematica Universitatis Comenianae, 42-43, 271-273.

Reay, J.R. and T.Zamfirescu (2000). Hamiltonian cycles in $T$-graphs. Discrete Computational Geometry, 24, 497-502. 
